03.1

\title{
Двойственность вихревой структуры, возникающей при сверхзвуковом обтекании области сопряжения затупленного тела и пластины вязким газом
}

\author{
(C) Е.В. Колесник ${ }^{1}$, А.А. Смирновский ${ }^{1,2}$, Е.М. Смирнов ${ }^{1}$ \\ ${ }^{1}$ Санкт-Петербургский политехнический университет Петра Великого, Санкт-Петербург, Россия \\ ${ }^{2}$ Физико-технический институт им. А.Ф. Иофрфе РАН, Санкт-Петербург, Россия \\ E-mail: kolesnik_ev@mail.ru
}

Поступило в Редакцию 5 марта 2020г.

В окончательной редакции 14 марта 2020 г.

Принято к публикации 18 марта 2020г.

Представлены результаты численного решения задачи сверхзвукового обтекания удлиненного цилиндрического тела, установленного на пластине, вдоль которой развивается пограничный слой. Рассматривается ламинарный режим обтекания при числе Маха внешнего потока, равном 6.7 , для двух значений числа Рейнольдса: $\operatorname{Re}=1.25 \cdot 10^{4}$ и $1.56 \cdot 10^{4}$. Дается анализ системы подковообразных вихрей, возникающих в области передней кромки тела. Установлено, что при обоих значениях Re существует два устойчивых решения, которые отвечают различным метастабильным состояниям потока. В основном решения различаются по числу вихрей в отрывной области и ее протяженности. При меньшем числе Рейнольдса оба решения являются стационарными, при большем значении Re одно из них сохраняет стационарность, а в другом присутствуют квазипериодические колебания.

Ключевые слова: численное моделирование, высокоскоростные течения, вязко-невязкое взаимодействие, подковообразные вихревые структуры, двойственность решения.

DOI: 10.21883/PJTF.2020.12.49519.18275

При обтекании затупленного тела, установленного на пластине, возникает сложная вихревая структура, характеризующаяся различной конфигурацией подковообразных вихрей. Большое число исследований посвящено изучению топологии вихревой структуры в зависимости от параметров течения для случая дозвукового обтекания $[1,2]$. В случае сверхзвукового обтекания возникающие скачки уплотнения приводят к сложным эффектам вязко-невязкого взаимодействия, что существенно влияет на вихревую структуру. Задачи данной направленности, важные как в теоретическом плане, так и для практических приложений, например, в аэрокосмической отрасли, также изучались многими исследователями (см., например, работы [3-7] и ссылки в них). Наиболее часто рассматривается модельная задача о сверхзвуковом обтекании вязким газом затупленного тела в виде ориентированной по потоку толстой пластины со скругленной передней кромкой. В частности, в расчетно-экспериментальной работе [4] для этой модельной конфигурации изучалась структура ламинарного потока перед обтекаемым телом для трех значений числа Рейнольдса, построенного по диаметру затупления: $\operatorname{Re}_{D}=1.25 \cdot 10^{4}, 2.50 \cdot 10^{4}, 3.75 \cdot 10^{4}$. Для всех значений $\operatorname{Re}_{D}$ авторы работы [4] трактовали полученные численные решения как стационарные и единственно возможные. Позднее в расчетном исследовании [5] было показано, что решение уже при $\operatorname{Re}_{D}=2.50 \cdot 10^{4}$ является нестационарным.
Цель настоящей работы - прояснить возможность возникновения двойственности и/или нестационарности численного решения задачи вязко-невязкого взаимодействия для той же модельной конфигурации при значениях числа $\operatorname{Re}_{D}$ в интервале от $1.25 \cdot 10^{4}$ до $2.50 \cdot 10^{4}$. В известной мере работа является продолжением исследований авторов [8], в которых при меньших числах Рейнольдса изучалось влияние числа Маха, протяженности пластины и температурного фактора на структуру потока и теплообмен.

Расчеты проводились в двух постановках: для половины рассматриваемой конфигурации (расчетная область показана на рис. 1) и в полной расчетной области без условия симметрии в срединной плоскости $X Z$. Согласно используемой модели вязкого совершенного газа, течение определяется следующим набором безразмерных параметров: числом Маха набегающего потока $\mathrm{M}$, числом Рейнольдса $\mathrm{Re}_{D}$, построенным по диаметру затупления $D$, числом Прандтля $\mathrm{Pr}$, температурным фактором $T_{w} / T_{i n}\left(T_{i n}-\right.$ температура набегающего потока), показателем адиабаты $\gamma$, а также протяженностью пластины $L_{\text {plate }}$ до места сопряжения. Решения задачи находились для набора параметров, соответствующего данным работы [4]: $\mathrm{M}=6.7, T_{w} / T_{i n}=4.75$, $\operatorname{Pr}=0.7, \gamma=1.4, L_{\text {plate }}=145 \mathrm{~mm}$. Расчеты проведены при двух значениях числа Рейнольдса: $\operatorname{Re}_{D}=1.25 \cdot 10^{4}$ и $1.56 \cdot 10^{4}$ (варьировалось за счет изменения $D$ от 2.5 до $3.125 \mathrm{~mm}$ ). На входной границе расчетной области задавался однородный поток, на поверхности тела и 


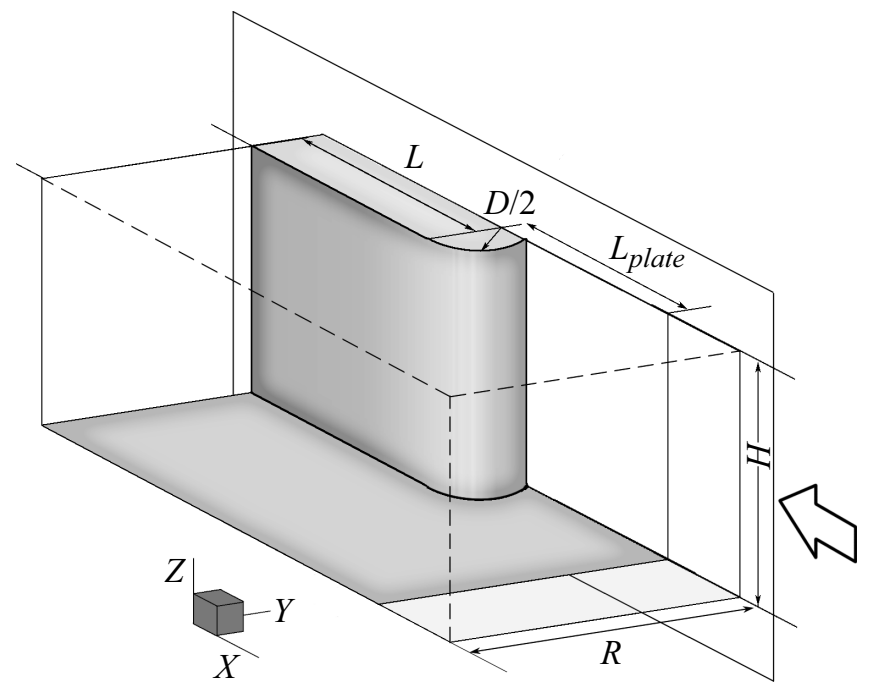

Рис. 1. Расчетная область.

пластине - условие прилипания. Для поверхности тела и пластины поддерживалась постоянная температура $T_{w}$.

Для расчетов (на основе нестационарной постановки) использовался конечно-объемный „неструктурированный“ программный код SINF/Flag-S, разрабатываемый в ИПММ СПбПУ; детали численной схемы даны в [9].

В случае расчетной области с наложенным условием симметрии сначала использовалась квазиструктурированная сетка, содержащая $10 \cdot 10^{6}$ ячеек. Затем в целях проверки сеточной сходимости для той же области расчеты были выполнены и на сетке с $25 \cdot 10^{6}$ ячеек. Расчеты для „полной“ области выполнялись на сетке, содержащей $20 \cdot 10^{6}$ ячеек. Для проведения расчетов использовались вычислительные ресурсы суперкомпьютерного центра СПбПУ [10].

Предыдущие исследования (см., например, работы [3-5]) показали, что при обтекании сверхзвуковым потоком установленного на пластине затупленного тела встречный градиент давления приводит к отрыву пограничного слоя. Внутри отрывной области возникает возвратное течение со сверхзвуковыми скоростями (числа Маха составляют около трети числа Маха набегающего потока), а также местные волны уплотнения, что приводит к повторному отрыву пристенного течения, и в результате перед телом формируется протяженная отрывная область, содержащая совокупность подковообразных вихрей.

В результате расчетов, стартовавших с разных начальных условий, было установлено, что при $\operatorname{Re}_{D}=1.25 \cdot 10^{4}$ существует два устойчивых стационарных решения, которые отвечают двум метастабильным состояниям потока с различной конфигурацией вихревой структуры (Solution 1 и Solution 2 на рис. 2,a).

Первое решение характеризуется более короткой отрывной областью, центр основного подковообразного вихря удален от передней кромки тела на расстояние около одного калибра $(D)$. Во втором решении отрывная область более протяженная, а центр основного вихря расположен почти в 2 раза дальше от обтекаемого тела. Кроме того, число вихрей во втором решении на единицу меньше (4 против 5).

Первое решение при данном значении $\operatorname{Re}_{D}=1.25 \cdot 10^{4}$ находится непосредственно при расчете с однородных полей искомых величин, построенных по значениям параметров потока на входе в расчетную область. Оно же устанавливается и при задании в качестве начальных условий решения, полученного при существенно меньшем числе Рейнольдса, в частности для уменьшенного в 5 раз числа Рейнольдса $\left(\operatorname{Re}_{D}=0.25 \cdot 10^{4}\right.$, старт с однородных полей). Отметим также, что в решении, полученном при $\operatorname{Re}_{D}=0.25 \cdot 10^{4}$ (на рисунках не показано), центр основного вихря располагается примерно на том же расстоянии от тела, что и в первом решении (Solution 1), представленном на рис. 2, a. Решение второго вида устанавливается при выборе в качестве начальных условий мгновенного поля нестационарного решения, развивающегося при старте с однородных полей при заметно большем числе Рейнольдса. Конкретно в настоящей работе это „вспомогательное“ нестационарное решение было получено для $\mathrm{Re}_{D}=2.50 \cdot 10^{4}$, при этом структура осредненного поля течения для данного решения аналогична представленной на рис. 2, $a$ для второго (стационарного) решения в случае $\operatorname{Re}_{D}=1.25 \cdot 10^{4}$. Задача поиска возможных точек ветвления или поворота стационарных решений (устойчивых и неустойчивых) в настоящей работе не ставилась. Исследование этих вопросов, включая прояснение возможности гистерезиса, требует весьма большого объема вычислений и составит предмет отдельной работы.

Возвращаясь к рассмотрению особенностей полученных решений, следует отметить, что распределение относительного теплового потока вдоль линии симметрии на пластине (рис. 2,b) помимо глобального максимума (находится вне поля рис. 2, $b$ ) содержит еще один ярко выраженный локальный максимум в первом решении и два локальных максимума меньшего уровня во втором решении. Последнее хорошо согласуется с численным решением и экспериментальными данными работы [4] (здесь следует отметить, что в [4] отсутствуют упоминания о возможности двойственности решения).

Оба решения сохраняют устойчивость и при расчете в полной постановке (без наложения условия симметрии). Измельчение сетки практически не сказалось на рассчитанных полях течения (рис. 2,b). В частности, для первого решения максимальные значения числа Маха возвратного течения (в нижней части основного вихря) при расчетах на исходной и измельченной сетках составили соответственно 33.1 и $33.5 \%$ от числа Маха набегающего потока $\mathrm{M}=6.7$.

При увеличенном до значения $1.56 \cdot 10^{4}$ числе Рейнольдса одно из решений (Solution 1) остается стационарным, а второе (Solution 2) является нестационар- 

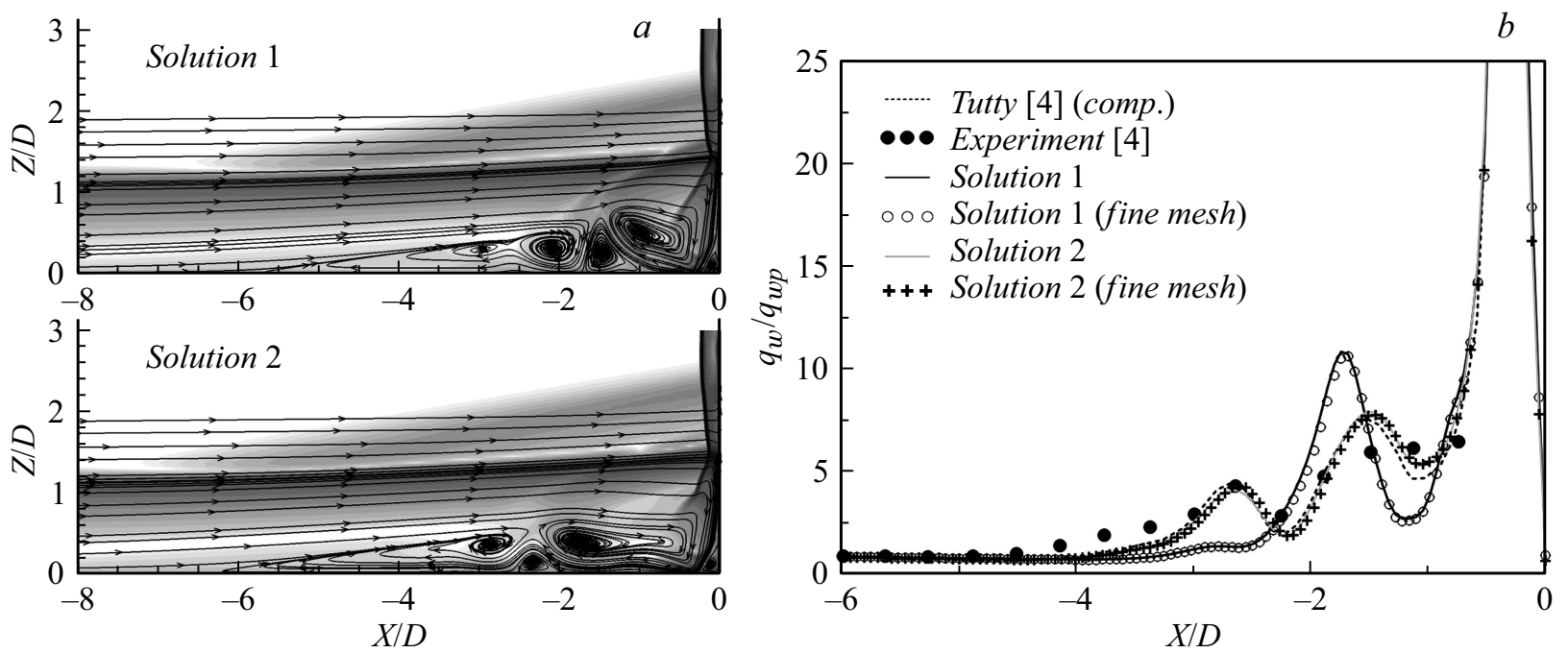

Рис. 2. Результаты расчетов при $\operatorname{Re}_{D}=1.25 \cdot 10^{4}$ для двух решений: $a$ - поле модуля градиента плотности и линии тока в плоскости симметрии; $b$ - распределение теплового потока вдоль линии симметрии.
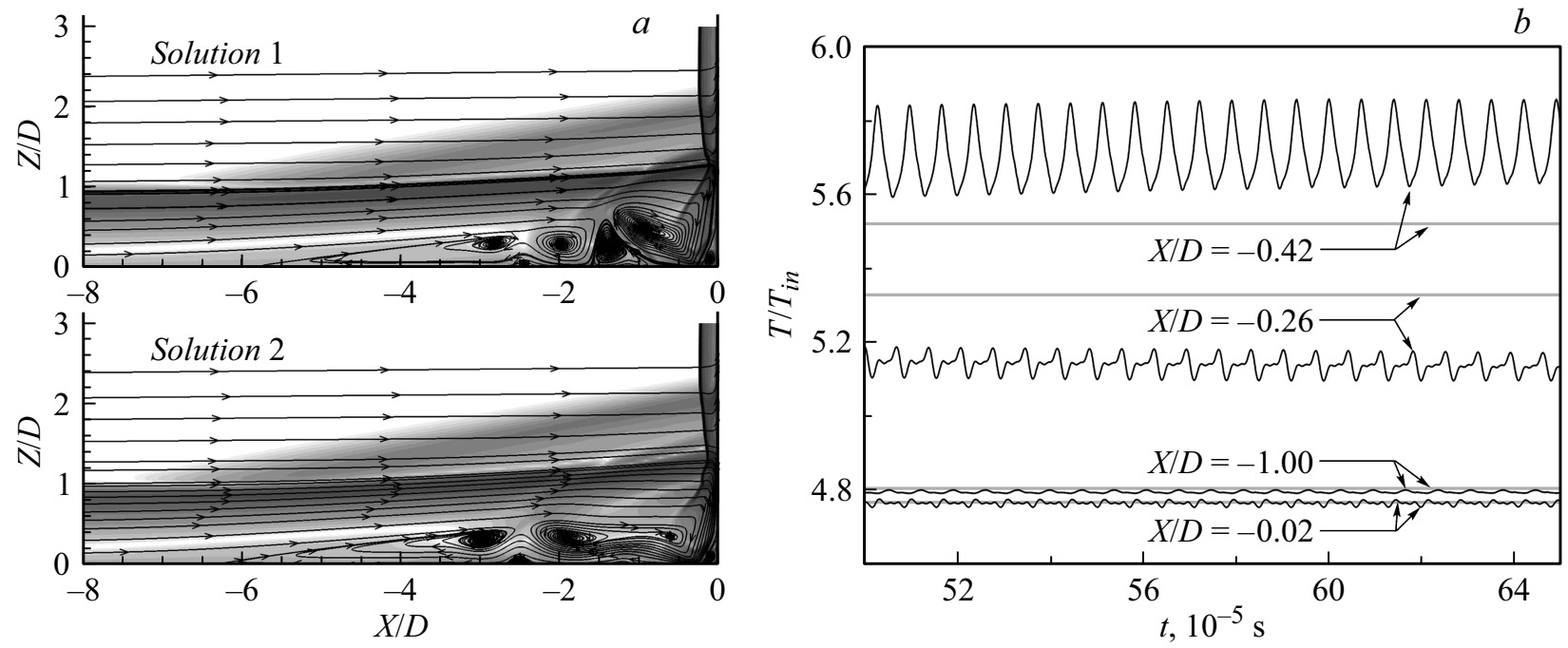

Рис. 3. Результаты расчетов при $\operatorname{Re}_{D}=1.56 \cdot 10^{4}$ для двух решений: $a$ - поле модуля градиента плотности и линии тока в плоскости симметрии (нестационарное решение усреднено по времени); $b$ - временнб́е изменения температуры в точках у поверхности пластины.

ным с устойчивыми квазипериодическими колебаниями потока, особенно сильными внутри отрывной области (рис. 3, a, для второго решения приведено осредненное по времени поле). Ранее [8] нами было показано, что увеличение длины отрывной области может сопровождаться переходом к нестационарному режиму течения. Поскольку в рассматриваемом здесь случае второму решению отвечает отрывная область большей протяженности, именно оно первым приобретает нестационарный характер при увеличении числа Рейнольдса.

На рис. 3, $b$ приведено значение относительной температуры $T / T_{\text {in }}$ в нескольких точках мониторинга у поверхности пластины (для сопоставления показаны также значения температуры для первого, стационарного, решения). Колебания с наибольшей амплитудой наблю- даются вблизи обтекаемого тела, в области глобального максимума теплового потока, а при $X / D<-0.8$ колебания незначительны. Конфигурация отрывной области, ее протяженность и толщина практически не меняются во времени.

Проведено трехмерное численное моделирование сверхзвукового потока вязкого газа при его взаимодействии с установленным на обтекаемой поверхности затупленным телом в виде ориентированной по потоку толстой пластины со скругленной передней кромкой. Численные решения получены при числе Маха 6.7 и двух значениях числа Рейнольдса.

Обнаружено, что при значении числа Рейнольдса $\operatorname{Re}_{D}=1.25 \cdot 10^{4}$ существует два устойчивых стационарных решения, которые отвечают двум метастабильным 
состояниям потока с различной конфигурацией вихревой структуры. Первое решение характеризуется менее протяженной отрывной областью и расположением центра основного подковообразного вихря на расстоянии около одного калибра от передней кромки тела; во втором решении центр основного вихря расположен существенно дальше от обтекаемого тела. Второе решение находится в хорошем согласии с экспериментальными и расчетными данными работы [4].

Показано также, что при увеличении числа Рейнольдса до значения $1.56 \cdot 10^{4}$ одно из двух решений остается стационарным, а второе является нестационарным и характеризуется устойчивыми квазипериодическими колебаниями потока, особенно сильными внутри отрывной области.

\section{Конфликт интересов}

Авторы заявляют, что у них нет конфликта интересов.

\section{Список литературы}

[1] Younis M.Y., Zhang H., Mehmood S., Aslam J. // Sci. China Technol. Sci. 2014. V. 57. P. 1342-1351.

[2] Launay G., Mignot E., Riviere N., Perkins R. // J. Fluid Mech. 2017. V. 830. P. 257-299.

[3] Schuricht P.H., Roberts G.T. // AIAA J. 1998. V. 1579. P. 1-9.

[4] Tutty O.R., Roberts G.T., Schuricht P.H. // J. Fluid Mech. 2013. V. 737. P. 19-55.

[5] Zhuang Y.Q., Lu X.Y. // Procedia Eng. 2015. V. 126. P. 134138.

[6] Mortazavi M., Knight D.D. // AIAA SciTech Forum - 55th AIAA Aerospace Sciences Meeting. Grapevine, Texas, 2017. P. 1-9.

[7] Азарова О.А., Ерофеев А.В., Лапушкина Т.А. // Письма в ЖТФ. 2017. Т. 43. В. 8. C. 93-101.

[8] Колесник Е.В., Смирнов Е.М. // ЖТФ. 2020. Т. 90. В. 2. C. $185-192$.

[9] Колесник E.В., Смирнов Е.М., Смирновский А.A. // Науч.техн. ведомости СПбГПУ. Физ.-мат. науки. 2019. Т. 12. № 2. C. 7-22. DOI: 10.18721/JPM.12201

[10] scc.spbstu.ru 